\title{
The Motion Evaluation of Front-Crawl Swimming upon Reaching the Goal on Unilateral Forearm-Amputee Swimmer
}

\author{
Tetsuro Tanigawa ${ }^{1}$, Kazumasa Kumamoto ${ }^{2}$, Hiroe Kataoka ${ }^{3}$, Takenori Awatani ${ }^{4}$, \\ Ikuhiro Morikita ${ }^{5}$ \\ ${ }^{1}$ Faculty of Education, Osaka Kyoiku University, Osaka, Japan \\ ${ }^{2}$ Faculty of Business Administration, Kindai University, Osaka, Japan \\ ${ }^{3}$ Faculty of Judo Therapy, Tokyo Ariake University of Medical and Health Sciences, Tokyo, Japan \\ ${ }^{4}$ Faculty of Physical Education, Kyushu Kyoritsu University, Fukuoka, Japan \\ ${ }^{5}$ Faculty of Sports Medicine Graduate School, Osaka University of Health and Sports Sciences, Osaka, Japan \\ Email: ^tanigawa@cc.osaka-kyoiku.ac.jp
}

How to cite this paper: Tanigawa, T., Kumamoto, K., Kataoka, H., Awatani, T., \& Morikita, I. (2018). The Motion Evaluation of Front-Crawl Swimming upon Reaching the Goal on Unilateral Forearm-Amputee Swimmer. Advances in Physical Education, 8, 116-128.

https://doi.org/10.4236/ape.2018.81012

Received: January 16, 2018

Accepted: February 10, 2018

Published: February 13, 2018

Copyright (c) 2018 by authors and Scientific Research Publishing Inc. This work is licensed under the Creative Commons Attribution International License (CC BY 4.0).

http://creativecommons.org/licenses/by/4.0/

\begin{abstract}
In this study, the finishing movement of front-crawl swimming upon reaching the goal was carried out by a unilateral forearm-amputee swimmer experimentally, and the difference between the finishing movements of the impaired side and the healthy side was compared in order to examine the effectiveness of each finishing movement. The subject was one female unilateral forearm-amputee swimmer (Impairment Classification S9) who has participated in Paralympic. The experiment practice was set that the subject carried out front-crawl swimming with the maximum effort without taking a breath from the $10 \mathrm{~m}$ point before the goal toward the goal wall. The practice conditions were set with four types, in order to verify with which arm the subject used to touch the goal wall (with the impaired side or the healthy side), and whether she touched the goal wall in the water or above the water. For evaluating a finishing movement, the standard value was set with the estimated time required when the swimmer touched the goal with her head top while maintaining the swimming speed when reaching the goal. And the difference between the standard time and the actual required time was calculated as the time loss (seconds). Among the four types of finishing movements evaluated in this research, the time loss during the finishing movement tends to decrease when the subject touched the goal with her healthy side in the water $(-0.20 \pm 0.01$ seconds), while it tends to increase when she touched the goal with her impaired side in the water $(0.08 \pm 0.07$ seconds). This result suggests the possibility that a unilateral forearm-amputee swimmer can expect better results by touching the goal with the healthy side in the water. For achieving
\end{abstract}


this, the swimmer needs to adjust his/her strokes as making the finishing touch by the healthy side in the water.

\section{Keywords}

Swimming, Touch the Goal Wall, Unilateral Forearm-Amputee Swimmer

\section{Introduction}

As the finishing movement of the front-crawl swimming in a competitive swimming race, it requires touching the goal wall with a part of a body. While swimming the front-crawl, the head of a swimmer would hit the wall if both arms were behind the head. For avoiding this, a swimmer carefully checks how many strokes he/she needs to touch the goal wall with his/her hand, while keeping the speed when reaching the goal. However, sometimes in a race, a swimmer can perform better swimming than he/she expected, and it results in faster swimming speed than usual. In this case, if the swimmer tries to carry out the usual finishing movement, the hand would touch the goal wall before stretching out the elbow, because he/she is getting to the goal wall too close at this point. This movement eventually results in slowing time, because of the bent elbow. On the other hand, when the swimming speed is slower than usual, due to bad condition or fatigue, the distance to the goal is getting longer than expected. In this case, if the swimmer carries out a finishing movement with keeping his/her arm forward without strokes, it may induce a slower pace and a poor record. As one could see, it is necessary for the swimmer to control the finishing movement of the front-crawl swimming in competitive swimming, when the goal wall is approaching.

For a swimmer with unilateral forearm-amputation impairment who has participated in the Paralympic swimming races (hereinafter referred to as a unilateral forearm-amputee swimmer), strokes made by the impaired side have weaker resistance against water, compared to the strokes by the healthy side. Lee et al. (2014) demonstrated that a stroke made by the healthy side of a unilateral forearm-amputee swimmer does not show a significant difference in its propulsive force, compared to a stroke by the dominant arm of a non-handicapped person. This research also suggests that there is a significant difference in the propulsive force between the impaired side and the healthy side of a unilateral forearm-amputee swimmer. When finishing movement is performed by the impaired side, the finishing action is carried out after the stroke made by the healthy side that produces equivalent propulsive force to that by a non-handicapped person. However, the swimmer touches the goal wall by the impaired side so that the timing of the finishing is delayed. On the other hand, when the finishing movement is done by the healthy side, the timing of touching the wall is not delayed, but the finishing is carried out after the stroke made by 
the impaired side with smaller propulsive force. Using computational fluid dynamics (CDF) model, Lecrivain et al. (2010) quantified the propulsive force in one stroke cycle of the impaired side of a unilateral forearm-amputee swimmer, and demonstrated that the slowdown resistance occurs on the impaired side during the latter half of the stroke. As this result suggests, when a unilateral forearm-amputee swimmer conducts the finishing movement, the factor slowing the time is included in either case. Thus, the finishing movement is extremely important for a swimmer with unilateral forearm-amputation impairment for improving the competitiveness, and it is clear that the finishing movement requires a highly advanced sporting skill. At the male S9 class $50 \mathrm{~m}$ freestyle swimming race in the 2016 Paralympics held in Rio de Janeiro, the difference between the time of the winning swimmer and that of the third-place swimmer was only 0.05 seconds (International Paralympic Committee, 2016). If an effective method for the finishing movement of front-crawl swimming by a unilateral forearm-amputee swimmer will be established, it will contribute to the methodology/coaching study of competitive swimming by handicapped swimmers and to improving the quality of the game.

Among the previous studies of the finishing movement, the finishing touch of butterfly stroke was evaluated and classified based on a waving movement, the number of kicking, and how to touch the goal wall in or out of the water (Tanigawa et al., 2012). In the case of evaluating a unilateral forearm-amputee swimmer, the finishing movement should be evaluated based on the four categories. In order to examine the effectiveness of each category, it is required to clarify which hand a swimmer uses to touch the goal, with the healthy side or the impaired side, and the place where the finishing touch is made, in the water or out of the water.

Moreover, Nomura (1997) evaluated the finishing movement during a swimming race. Firstly, the required swimming time between the $95 \mathrm{~m}$ to the $100 \mathrm{~m}$ points, and between the $75 \mathrm{~m}$ and the $95 \mathrm{~m}$ points were recorded respectively, and then compared to the actual time from the race during the finishing phase. When evaluating the finishing movement through the elapsed time in the $5 \mathrm{~m}$ between the $95 \mathrm{~m}$ point and the $100 \mathrm{~m}$ point, not only the finishing movement, but the influence caused by the change in the swimming speed should be considered. Thus when examining the finishing movement method, it is necessary to reduce the influence of the swimming speed as much as possible. Taking the aforementioned information into consideration, when focusing on the finishing movement and examining how the differences in finish movements affect the time records, it requires experiments to carry out the actual finishing movements.

In this study, finishing movements of front-crawl swimming by a unilateral forearm-amputee swimmer was experimentally performed. The purpose of this study is to compare the finishing movements of the impaired side and the healthy side, and verify the effective approaches in the finishing movements. 


\section{Method}

\subsection{Subject}

The subject was one female unilateral forearm-amputee swimmer (Impairment Classification S9) who has participated in the Paralympic race. For this reason, she has the stable finishing movements skills. The classification was classified by the Classifiers belong the International Paralympic Committee (2011) Swimming according to the Classification Rules and Regulations. Her height was 1.66 $\mathrm{m}$, weight was $59.0 \mathrm{~kg}$, the distance of the vertical component between the amputation stump of the impaired side and the head top was $0.08 \mathrm{~m}$ (when the shoulder joint is stretched to 180 degrees) and the distance of the vertical component between the middle fingertip of the healthy side and the head top was $0.38 \mathrm{~m}$ (when the shoulder joint is stretched to 180 degrees). Her best time in the $50 \mathrm{~m}$ freestyle was 31.13 seconds. An informed consent was obtained from the subject regarding the experiment contents prior to the experiment. Also, this research was approved by the ethic committee of Kyushu Kyoritsu University.

\subsection{Experiment Practice}

The experiment practice was set that the subject carried out front-crawl swimming with the maximum effort without taking a breath from the $10 \mathrm{~m}$ point toward the goal wall. In order to allow the subject to gain propulsive force from the starting point, a temporary wall (Turn Master by FINIS, Inc.) was installed at the $10 \mathrm{~m}$ point. The practice conditions were; 1 ) the healthy side touches the goal wall in the water (hereinafter referred to as "healthy side touch in the water", Figure 1(a)), 2) the impaired side touches the goal wall above the water (hereinafter referred to as "impaired side touch above the water", Figure 1(b)), 3) the impaired side touches the goal wall in the water (hereinafter referred to as "impaired side touch in the water", Figure 1(c)), and 4) the healthy side touches the goal wall above the water (hereinafter referred to as "healthy side touch above the water", Figure 1(d)). Before the experiment was conducted, the subject practiced each finishing touch several times. Afterward, each finishing touch was conducted on a random for four times, or 16 times in total, while taking an adequate rest in between.

\subsection{Recording Method}

A video camera (GC-YJ40, FPS 60 frames/seconds, JVC Kenwood) was laterally installed to the moving direction of the subject in order to record the range from the goal wall to the $6 \mathrm{~m}$ point. In addition, an underwater camera (Hero 5 Black, FPS 60 frames/seconds, GoPro) was installed inside the pool with a fixed angle. The video camera filmed the process of in-and-out of the water finishing touches by both the impaired side and the healthy side, and the underwater camera filmed the moments of finishing touches in the water. The video camera and the underwater camera were synchronized using a synchronous light-emitting device (By Sports Sensing). 


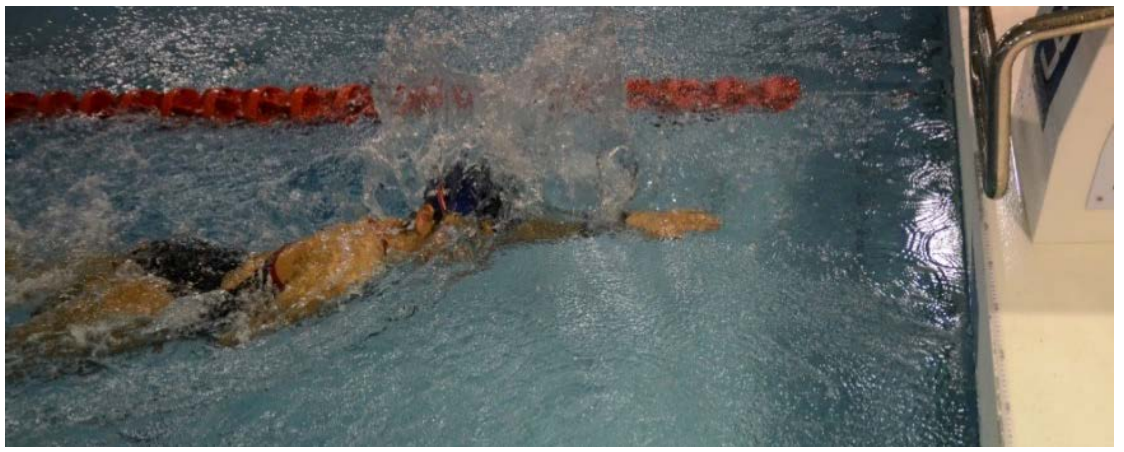

(a)

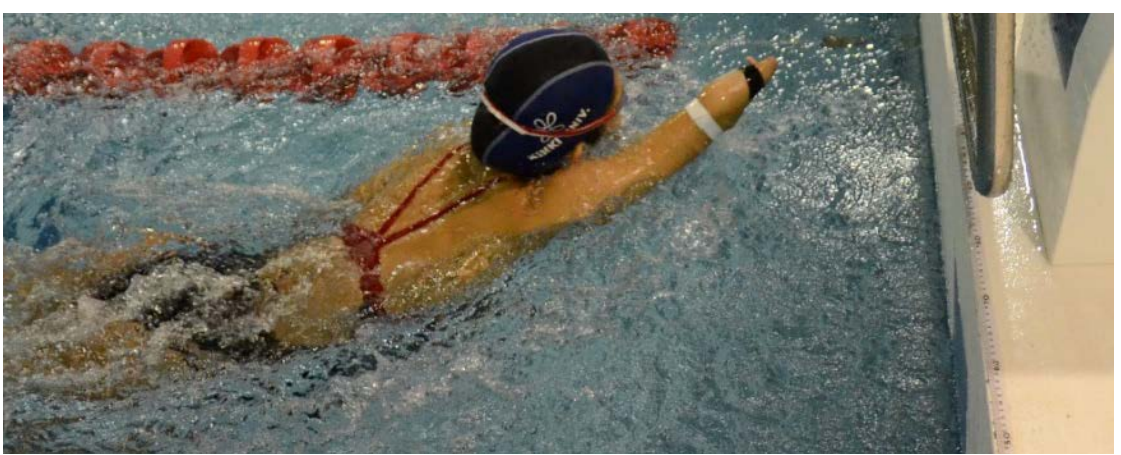

(b)

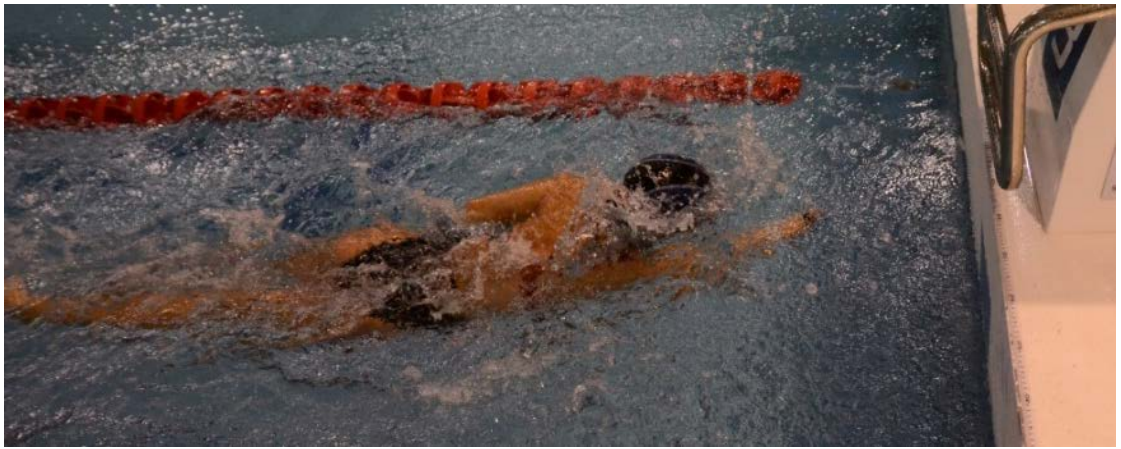

(c)

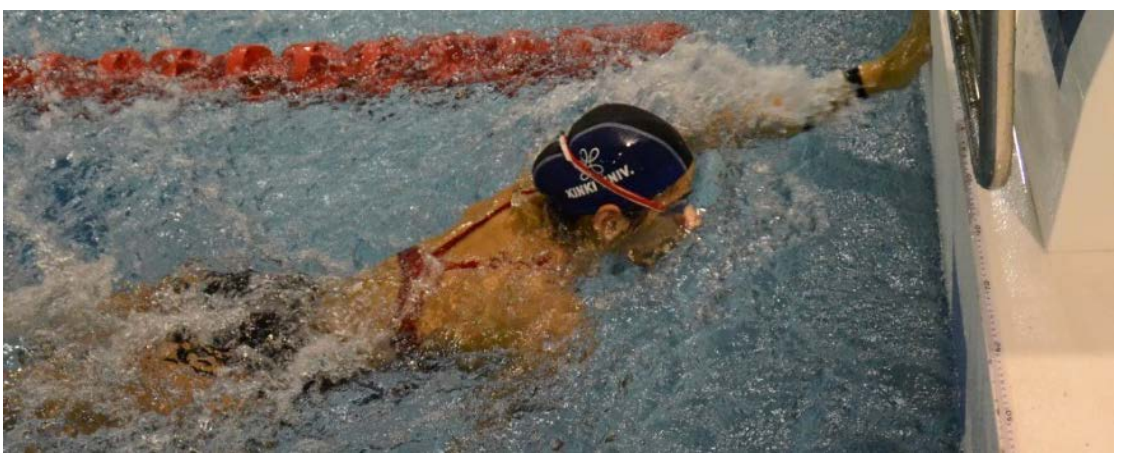

(d)

Figure 1. (a) The healthy side touches the goal wall in the water (healthy side touch in the water). (b) The impaired side touches the goal wall above the water (impaired side touch above the water). (c) The impaired side touches the goal wall in the water (impaired side touch in the water). (d) The healthy side touches the goal wall above the water (healthy side touch above the water). 


\subsection{Data Processing}

For the data analysis, the footages obtained by the video camera were captured into a personal computer using a motion analysis software (Frame Dias-V, $\mathrm{DKH}$ ). The reference markers were attached on the head top, the fingertips of the healthy side, the amputation stamp and the other six places. The data captured through these markers were manually digitized at 60 frames per second. On the two-dimensional coordinates obtained from the aforementioned body parts, the traveling direction of the subject was set on the $\mathrm{Y}$ axis direction. And the axis perpendicular to $\mathrm{Y}$ axis on the horizontal plane was set as $\mathrm{X}$ axis. They were converted to true length based on the reference markers using two-dimensional DLT method.

\subsection{Categorization of Phases}

The moment when the fingertips of the healthy side went into the water by a final stroke was set as the standard point. And the duration between the standard point to the moment when a part of the subject's body touched the goal was set as the finishing phase (Figure 2). The interval from the standard point to the 6 $\mathrm{m}$ points away from the goal was set as the swimming area.

\subsection{Measurement Items}

From the digitized data, the average swimming speed in the swimming area was calculated, and the average value of the 16 practices was calculated as the swimming speed ( $\mathrm{m} /$ seconds). Furthermore, the $\mathrm{Y}$ axis distance between the goal wall and the head top at the moment the fingertips of the healthy side touched the water by the final stroke before touching the goal was calculated as the distance in the finishing phase (m). In addition, the required time of the finishing phase was calculated (seconds).

\section{Touching on the goal wall}

The $6 \mathrm{~m}$ point

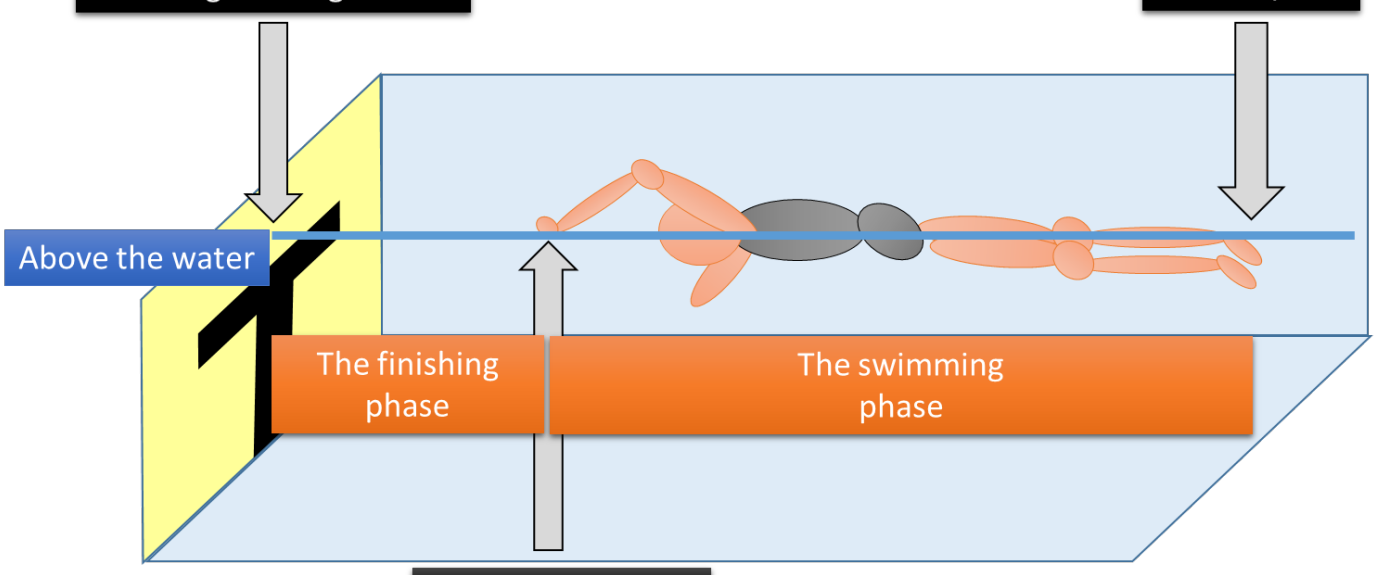

The standard point

The point where the healthy side arm goes into the water

Figure 2. The Classification of the finish phase. 


\subsection{Evaluation Method}

The evaluation of the finishing movement was set as the estimated required time of the finishing phase, which was calculated by dividing the distance in the finishing phase with the swimming speed. The difference between this estimated required time of the finishing phase and the actual time required during the finishing phase was calculated as the time loss (seconds). When the time loss was plus, it means that touching the wall with the amputated stump of the impaired side or the hand of the healthy side took more time than touching it with the head top. When it was minus, it means that touching the wall with the amputated stump of the impaired side or the hand of the healthy side took less time than touching it with the head.

\subsection{Statistics}

The relationship between the distance in the finishing phase and the time loss was tested using a two-tailed Pearson's test. Statistical significance was set at $p<$ 0.05 .

\section{Result}

Table 1 shows the results of the swimming speed, the required time in the finishing phase, the time loss and the distance in the finishing phase of the four types by four practices of finishing movements conducted for this study. Furthermore, Figure 3 shows the relationship between the distance in the finishing phase and the time loss (The healthy side touch in the water: $\mathrm{r}=0.690, p>0.05$, The impaired side touch above the water: $\mathrm{r}=-0.043, p>0.05$, The impaired side touch in the water: $\mathrm{r}=-0.299, p>0.05$, The healthy side touch above the water: $\mathrm{r}=-0.648, p>0.05)$.

The average of the swimming speed and its standard deviation was $1.39 \pm 0.03$ $\mathrm{m} / \mathrm{seconds}$ in all of 16 practices. When swimming $50 \mathrm{~m}$ in this speed, it would finish in 36.00 seconds, though it does not include the time required for diving into the water.

Regarding the subject of this study, the vertical component distance between the middle fingertip of the healthy side and the head top of was $0.38 \mathrm{~m}$. The vertical component distance between the amputated stump and the head top was $0.08 \mathrm{~m}$. The average swimming speed was $1.39 \mathrm{~m} /$ seconds in this study. Compared to touching the goal with the head top, touching the goal wall with the middle fingertip of the healthy side resulted 0.20 seconds faster, and touching it with the amputated stump resulted 0.05 seconds faster. In other words, if only the length of the arms are taken into consideration, this means that touching the goal wall with either of the healthy or the impaired hand took less time than touching it with the head top.

Next, regarding the average and standard deviation of the time loss of each practice, the practices that touched the wall with the healthy side (in the water: $-0.20 \pm 0.01$ seconds, above the water: $-0.17 \pm 0.04$ seconds) tended to be less 
Table 1. The results of the swimming speed, the required time in the finishing phase, the time loss and the distance in the finishing phase.

\begin{tabular}{|c|c|c|c|c|c|}
\hline $\begin{array}{l}\text { The Category of } \\
\text { finishing } \\
\text { movements }\end{array}$ & $\begin{array}{c}\text { The } \\
\text { practice }\end{array}$ & $\begin{array}{l}\text { The } \\
\text { swimming } \\
\text { speed } \\
\text { (m/seconds) }\end{array}$ & $\begin{array}{l}\text { The required time } \\
\text { in the finishing } \\
\text { phase (seconds) }\end{array}$ & $\begin{array}{l}\text { The time loss } \\
\text { (seconds) }\end{array}$ & $\begin{array}{c}\text { The distance } \\
\text { in the finishing } \\
\text { phase }(\mathrm{m})\end{array}$ \\
\hline \multirow{4}{*}{$\begin{array}{l}\text { The healthy side } \\
\text { touch in the } \\
\text { water }\end{array}$} & 1 & 1.36 & 0.60 & -0.26 & 0.84 \\
\hline & 2 & 1.42 & 0.78 & -0.16 & 0.99 \\
\hline & 3 & 1.41 & 0.95 & -0.16 & 1.14 \\
\hline & 4 & 1.40 & 0.38 & -0.23 & 0.51 \\
\hline \multirow{6}{*}{$\begin{array}{c}\text { The impaired side } \\
\text { touch above the } \\
\text { water }\end{array}$} & M. \pm S.D. & $1.39 \pm 0.03$ & $0.68 \pm 0.24$ & $-0.20 \pm 0.05$ & $0.87 \pm 0.27$ \\
\hline & 1 & 1.35 & 1.08 & 0.01 & 1.10 \\
\hline & 2 & 1.35 & 1.15 & 0.00 & 1.20 \\
\hline & 3 & 1.39 & 1.05 & -0.01 & 1.11 \\
\hline & 4 & 1.37 & 1.00 & 0.00 & 0.97 \\
\hline & M. \pm S.D. & $1.36 \pm 0.02$ & $1.07 \pm 0.06$ & $0.00 \pm 0.01$ & $1.10 \pm 0.09$ \\
\hline \multirow{5}{*}{$\begin{array}{l}\text { The impaired } \\
\text { side } \\
\text { touch in the } \\
\text { water }\end{array}$} & 1 & 1.35 & 1.17 & 0.00 & 1.29 \\
\hline & 2 & 1.38 & 1.17 & 0.08 & 1.16 \\
\hline & 3 & 1.38 & 0.93 & 0.08 & 0.84 \\
\hline & 4 & 1.46 & 1.22 & 0.16 & 1.15 \\
\hline & M. \pm S.D. & $1.39 \pm 0.05$ & $1.12 \pm 0.13$ & $0.08 \pm 0.07$ & $1.11 \pm 0.19$ \\
\hline \multirow{5}{*}{$\begin{array}{l}\text { The healthy side } \\
\text { touch above the } \\
\text { water }\end{array}$} & 1 & 1.44 & 0.98 & -0.19 & 1.29 \\
\hline & 2 & 1.38 & 1.08 & -0.12 & 1.27 \\
\hline & 3 & 1.40 & 1.02 & -0.21 & 1.37 \\
\hline & 4 & 1.40 & 0.95 & -0.17 & 1.24 \\
\hline & M. \pm S.D. & $1.40 \pm 0.02$ & $1.01 \pm 0.06$ & $-0.17 \pm 0.04$ & $1.29 \pm 0.05$ \\
\hline
\end{tabular}

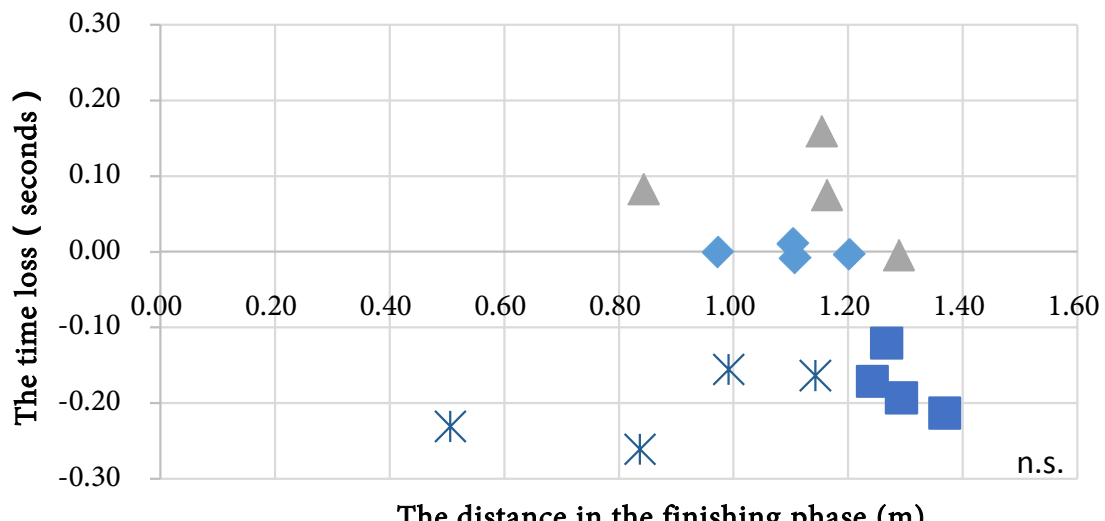

The distance in the finishing phase (m)

$\checkmark$ The impaired side touch above the water $\Delta$ The impaired side touch in the water

The healthy side touch above the water $\quad *$ The healthy side touch in the water

Figure 3. The relation between the distance in the finishing phase and the time loss.

time loss compared to the practices that touched the wall with the impaired side (in the water: $0.08 \pm 0.01$ seconds, above the water: $0.00 \pm 0.01$ seconds). This indicates that conducting the finishing movement with the healthy side requires 
shorter time.

As shown by the comparative analysis of the healthy side touch in the water, even if the distance in the finishing phase was the longest $(1.14 \mathrm{~m})$, the time loss $(-0.16$ seconds) was less than that of the impaired side touch in the water (distance: $1.15 \mathrm{~m}$, time loss: 0.16 seconds) or the impaired side touch above the water (distance: $1.11 \mathrm{~m}$, time loss: -0.01 seconds) with the shortest distance in the finishing phase.

Moreover, regarding the average and the standard deviation of the distance in the finishing phase among the four types of finishing movements, the healthy side touch in the water tended to be the shortest $(0.87 \pm 0.27 \mathrm{~m})$. There was no difference between the impaired side touch in the water $(1.10 \pm 0.08 \mathrm{~m})$ and the impaired side touch above the water $(1.11 \pm 0.19 \mathrm{~m})$, and the healthy side touch above the water tended to be the longest $(1.29 \pm 0.05 \mathrm{~m})$.

\section{Discussion}

\subsection{Effectiveness of Each Finishing Movement}

As the results of this study show, the healthy side touch in the water has the least time loss and the impaired side touch in the water has the most time loss. When touched the goal wall by the healthy side in the water, a stroke was made by the impaired side just before touching the goal. As shown in a previous study (Lecrivain et al., 2010), there are cases where decelerating force occurred during the latter half of the stroke. In case of the distance to the goal wall being long, the swimmer would lose the speed significantly before touching the wall and consequently the stroke by the impaired side is faster. However, regarding the result of the healthy side touch in the water, considering the distance from the fingertips of the healthy side to the head top, it is clear that touching the wall with the fingertips of the healthy side reaches the goal 0.20 seconds faster compared to touching the wall with the head top. This is an equivalent result to the time loss of the healthy side touch in the water $(-0.20 \pm 0.05$ seconds). This outcome suggests that the results of the healthy side touch in the water are related to the length of the arm.

Moreover, the time loss of the healthy side touch above the water is greater than that of the healthy side touch in the water. It shows the possibility to reach the goal faster than conducting the finishing movement with the head top. Even in a situation where the finishing movement needs to be conducted with the impaired side, the swimmer has more chance to finish faster if the swimmer strokes on the impaired side and conducts the finishing movement with the healthy side even if the elbow is not stretched out completely. This finishing movement method may be adopted as the second option, in case that the healthy side touch in the water is difficult to make.

On the other hand, the impaired side touch in the water tends to produce more time loss, compared to either healthy side touch in the water. Even though considering the length of the arm, it can be theorized to make 0.05 seconds faster 
finish than the head top. The results of this study showed that it was slower than touching the wall with the head top (impaired side in the water: $0.08 \pm 0.07$ seconds, impaired side above the water $0.00 \pm 0.01$ seconds). In case of the impaired side touch in the water, it was conjectured that the last stroke before finishing is done by the healthy side, therefore, the propulsive force is unlikely to decrease. However, the actual result differed from the prediction. As shown in Figure 4, the head was above the water by the moment when the impaired side touched the wall. This is thought to be the reason of this result. If the head was above the water, the body was lifted and received more resistance as the underwater cross-sectional area increased. The reason for this movement is that when touching the goal wall by the impaired side, the swimmer could not stop with the strength of the impaired side arm alone. Therefore the subject unconsciously prevented the head that was close to the tip of the impaired side from hitting the wall.

\subsection{The Relationship between Each Finishing Movement and Distance in the Finishing Phase}

The result of this study shows that the healthy side touch in the water tends to have shorter distance in finishing phase with wider range, compared to the three other finishing movements. Thus, if the last point where the healthy side goes into the water is getting closer to the wall, it is easier to make the healthy side touch in the water.

When comparing the healthy side touch with the longest distance to the goal wall, the time loss was still shorter than the either case of the impaired side touch in the water or above the water with the closest distance in the finishing phase. These results suggest the necessity of conducting the finishing movement on the healthy side. However, in order to conduct the healthy side touch in the water, a swimmer has to adjust the stoke speed before the finishing movement. He/she is required to make the last stroke with his/her healthy hand, with adjusting the point where the last stroke goes into the water to the most ideal position. By the time the race goes into the final phase where the finishing movement is

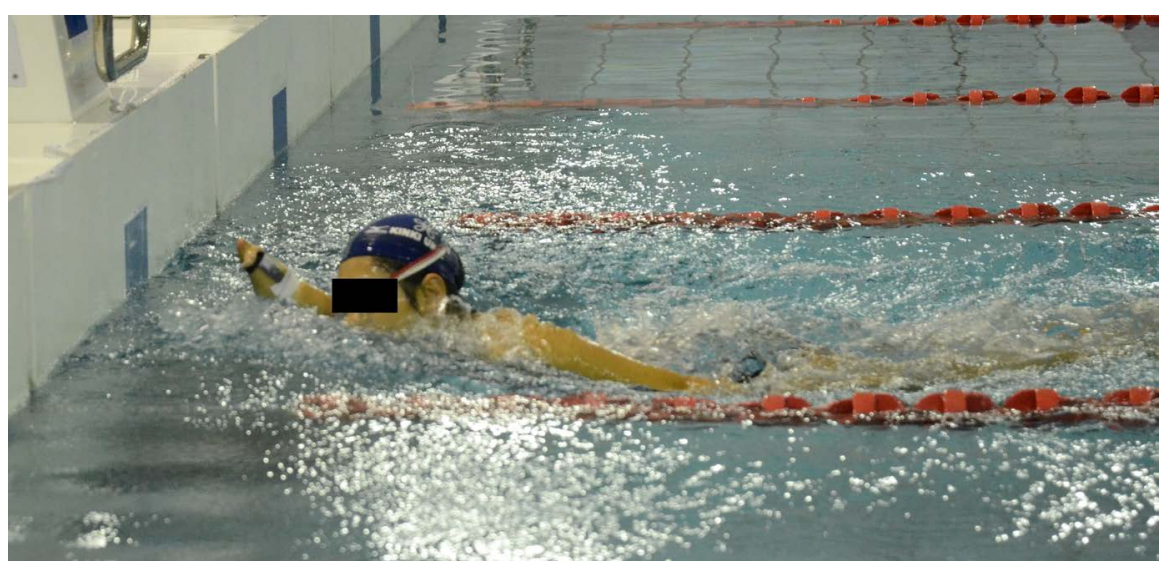

Figure 4. When reaching to the goal wall with the impaired side. 
conducted, the swimmers are utterly exhausted, and it is likely to be difficult for them to quicken their strokes. Okuno et al. (1999) demonstrated from the analysis results of the Japan Championship Race that the group that got through to the finals quickened their pace at the last phase of the race compared to the groups that were eliminated at the preliminaries. In other word, the athletes with higher capabilities can both quicken and slow down their pace even in the final phase of a race. Thus, it can be conjectured that they can adjust their strokes easier than for the swimmers who are fatigued. As one could see, since the finishing movement is conducted in the final phase of a race, a swimmer needs to perform the goal action as preventing the time loss during actual races. Therefore, it is necessary to acquire swimming skill and stamina that can control exhaustion, in order to make an approach to the goal while adjusting strokes, and perform the healthy side touch in the water. If it appears that the finishing movement is about to be conducted by the impaired side, it is better to either keep the healthy side forward or make one more stroke to achieve the healthy side touch above the water.

\subsection{Limited Range of This Study}

In this study, the time loss was used as the parameter to indicate the success and failure of the finishing touch. The subject was asked to swim at the finishing phase speed, and the evaluation was made by the comparison of the differences between the required time for touching the goal wall with the head top and the actual finishing phase. Takagi et al. (1997) demonstrated that the underwater resistance generated when a Japanese swimmer is in the streamline position increases proportionally to the 1.91 power of the propulsive force. This shows that a practice with higher propulsive force has more speed loss due to the resistance, compared to a practice with lower propulsive force. In case that the practices require more time to keep one arm remained forward, and the swimming speeds vary more depending on finishing movements, the swimming speed can be considered to be a confounding factor that causes time loss. The subject of this study showed $1.39 \pm 0.03 \mathrm{~m} / \mathrm{seconds}$. However, regarding the average and the standard deviation of the swimming speed in the study by Tanigawa et al. (2012), which evaluated the finishing movement of the butterfly stroke, the lowest standard deviation was $1.75 \pm 0.02 \mathrm{~m} / \mathrm{seconds}$, and the highest was $1.76 \pm 0.07 \mathrm{~m} / \mathrm{seconds}$. Compared to the previous studies, the variations in the swimming speed in this study are relatively small. Moreover, the difference between the best time by the subject of this study in the $50 \mathrm{~m}$ freestyle ( 31.13 seconds) and the time when she swam $50 \mathrm{~m}$ in the average swimming speed of the experiment practice $(36.00$ seconds) is relatively large. This result suggests the possibility that more time loss may occur in the actual competitive races.

\section{Conclusion}

The following three points were clarified by this study. 
1) Among the four types of finishing movements conducted for this study, the healthy side touch in the water tended to have the least time loss $(-0.20 \pm 0.01$ seconds), and the impaired side touch in the water tended to have the most time loss (0.08 \pm 0.07 seconds).

2) Regarding the average and the standard deviation of the distance in the finishing phase among the four types of finishing movement, the healthy side touch in the water tended to be the shortest $(0.87 \pm 0.27 \mathrm{~m})$, no difference between the impaired side touch in the water $(1.10 \pm 0.08 \mathrm{~m})$ and the impaired side above the water touch $(1.11 \pm 0.19 \mathrm{~m})$, and the healthy side above the water touch tended to be the longest $(1.29 \pm 0.05 \mathrm{~m})$.

3) Among the healthy side touch in the water conducted for this study, the time loss $(-0.16$ seconds) of the practice with the longest distance in the finishing phase $(1.14 \mathrm{~m})$ tended to be less than those of the impaired side touch in the water (distance: $1.15 \mathrm{~m}$, time loss: 0.16 seconds) and the impaired side above the water touch (distance: $1.11 \mathrm{~m}$, time loss: -0.01 seconds).

The results discussed in the above suggest that a unilateral forearm-amputee swimmer can expect better results from performing the finishing movement to touch the goal wall with the healthy side hand in the water. In order to achieve this, it requires for a swimmer to adjust strokes toward the goal as to touch the wall with the healthy side in the water. However, when the swimmer is too fatigued to adjust the strokes, it can be proposed to keep the healthy side forward without doing a stroke, for avoiding the finishing movement with the impaired side.

\section{Acknowledgements}

This study could be made with having the great support by the 2015 general research grant from the Japan Society of Coaching Studies. We deeply appreciate their great support extended to this research.

\section{References}

Lecrivain, G., Payton, C., Slaouti, A., \& Kennedy, I. (2010). Effect of Body Roll Amplitude and Arm Rotation Speed on Propulsion of Arm Amputee Swimmers. Journal of Biomechanics, 43, 1111-1117.

Lee, C. J., Sanders, R. H., \& Payton, C. J. (2014). Changes in Force Production and Stroke Parameters of Trained Able-Bodied and Unilateral Arm-Amputee Female Swimmers during a $30 \mathrm{~s}$ Tethered Front-Crawl Swim. Journal of Sports Sciences, 32, 1704-1711.

https://doi.org/10.1080/02640414.2014.915420

Nomura (1997). The Science of Backstroke. The Report of the Backstroke Research, 41-46.

Takagi, H., Nomura, T., Matsui, A., \& Minami, T. (1997). The Drag Coefficients of the Bodies under Water of Japanese Competitive Swimmers. International Journal of Sport and Health Science, 41, 484-491.

Okuno, K., Ikuta, Y., Honbe, Y., Ishikawa, M., Wakayoshi, K., \& Nomura, T. (1999). The Search for the Stroke Frequency Variation in Men's $5 \mathrm{~m}$ Freestyle Swimming-All Japan Championship in 1997. The Japanese Journal of Sport Methodology, 12, 17-24. 
International Paralympic Committee (2016). Rio 2016 Paralympic Games. https://www.paralympic.org/results/historical

International Paralympic Committee (2011). World Para Swimming Classification Rules and Regulations.

https://www.paralympic.org/sites/default/files/document/120706163426076_2011_05_3 $\underline{0 \_ \text {Swimming_Classification_Regulations.pdf }}$

Tanigawa, T., Hasegawa, H., Ikuta, Y., Kida, N., \& Nomura, T. (2012). What Kind of Goal Touch Is Effective in the Butterfly? Japan Journal of Coaching Studies, 27, 67-76. 